\title{
Tecnologías de la información y la comunicación, como estrategia pedagógica para fomentar el interés académico en estudiantes ${ }^{1}$
}

\section{Information and Communication Technologies, as a strategy to promote academic interest in students}

DOI: http://dx.doi.org/10.17981/cultedusoc.9.3.2018.49

Artículo de investigación. Fecha de recepción: 15/06/2018. Fecha de aceptación: 27/11/2018

Suleyma Dávila-García;

Elinor Lobo-Galán; German Belmonte-Castañeda; Hernando Mercado-Sanjuan; John Saent-Gil; Juan Martínez-Uzeta; María Camargo-Páez; Martha De La Hoz-Martínez; Mónica Osorio-Machado; Silvia Córdoba-Munño; Yaneth Pulgar-Barone; Yasmin Buelvas-Gutiérrez; Yecenia Marriaga-Pertuz; Eduardo Hoyos-Manotas; Alba Villazón-De la Cruz; Ana Escorcia-Angarita; Beatriz Navarro-Navarro; Rafaela Jimeno-Ortiz; Edinson Pertuz-Caballero; Luisa Orozco-Andrade; Mercedes Lobo-Batista; Mirian PallaresEscorcia; Rosa Hernández; Marta Vega Simanca; Nancy Villegas-Uribe; Rosiris Barros-Anaya; Tomasa Rodríguez-Vargas; Amarilis Díaz Granado; Margarita Báez-Cadavid y Stella De La Cruz-Barraja ${ }^{3}$ Institución educativa departamental de Fundación (Colombia) sudaga_69@hotmail.com

Para citar este artículo:

Dávila-García, S., Lobo-Galán, E., Belmonte-Castañeda, G., Mercado-Sanjuan, H., Saent-Gil, J., Martínez-Uzeta, J., Camargo-Páez, M., De La Hoz-Martínez, M., Osorio-Machado, M., Córdoba-Munño, S., Pulgar-Barone, Y., Buelvas-Gutiérrez, Y., Marriaga-Pertuz, Y., Hoyos-Manotas, E., Villazón-De la Cruz, A., Escorcia-Angarita, A., Navarro-Navarro, B., Jimeno-Ortiz, R., Pertuz-Caballero, E., Orozco- Andrade, L., Lobo-Batista, M., PallaresEscorcia, M., Hernández, R., Vega, M., Villegas-Uribe, N., Barros-Anaya, R., RodríguezVargas, T., Díaz, A., Báez-Cadavid, M. y De La Cruz-Barraja, S. (2018). Tecnologías de la información y la comunicación, como estrategia pedagógica para fomentar el interés académico en estudiantes. Cultura. Educación y Sociedad 9(3), 423-432. DOI: http://dx.doi. org/10.17981/cultedusoc.9.3.2018.49

\section{Resumen}

Teniendo en cuenta la incidencia de las nuevas tecnologías en la educación de hoy, el propósito de la presente investigación radica en implementar las tecnologías de la información y la comunicación TIC en el aula de clase para fomentar el interés académico en los estudiantes de la IED Fundación. Metodológicamente la investigación se orientó desde una mirada Cualitativa, bajo el paradigma sociocritico; la unidad de análisis estuvo constituida por cuarenta (40) estudiantes del grado octavo entre las edades de catorce (14) quince (15) y dieciséis (16) años; como técnica de recolección de información se utilizó la observación participante, como instrumento de recolección, el diario de campo; a partir de las actividades desarrolladas con la implementación de las TIC en el aula de clase se encontró que los estudiantes presentan mayores niveles de motivación e interés por aprender, mejorando significativamente su promedio de notas cuándo se trabajan los contenidos curriculares articulados con estas.

Palabras clave: Tecnologías de la información y la comunicación (TIC), estrategias pedagógicas, interés académico.

\section{Abstract}

Taking into account the incidence of new information and communication technologies in education today, the purpose of research in the implementation of information technology and communication students of the IED Foundation. Methodologically, the research was oriented from a Qualitative perspective, under the socio-critical paradigm; the analysis unit consisted of forty (40) eighth grade students between the ages of fourteen (14) fifteen (15) and sixteen (16) years; as a tool for gathering information, the collection instrument, the field diary; From the activities developed with the implementation of ICT in the classroom, students were found to have higher levels of motivation and interest in learning, significantly improving their grade point average when working with articulated curricular contents

Keywords: Information and communication technologies, pedagogical strategies, academic interest.

1 Este artículo ha sido derivado del Proyecto Fortalecimiento de la Cultura Ciudadana y Democrática en CT+I a través de la IEP apoyada en TIC en el Dpto. del Magdalena

2 Docente líder del grupo de investigación "Semillas de Paz".

3 Docentes de la institución educativa departamental de Fundación, del grupo de investigación "Semillas de Paz".

- The author; licensee Universidad de la Costa - CUC.

Cultura, Educación y Sociedad vol. 9 no. 3, pp. 423-432. Diciembre, 2018

Barranquilla. ISSN 2389-7724 Online 


\section{Introducción}

Durante los últimos años ha sido notorio los cambios y el desarrollos en las instituciones educativas; cambios evidenciados en las nuevas metodologías de enseñanza, en la reorganización de los procesos de pedagógicos, en la adquisición de nuevas tecnologías y el desarrollo de modelos de aprendizaje basados en competencias; transformaciones que se han impulsado con el objetivo de potencializar los procesos de enseñanzaaprendizaje y, por ende, de formar un estudiante integral capaz de enfrentarse los retos del mundo globalizado de hoy de manera eficaz, dinámica y holística (Marín, Inciarte, Hernández y Pitre, 2017).

Uno de los principales cambios en las instituciones es la incorporación de las nuevas tecnologías como elemento de innovación educativa en la práctica docente, ya que como lo expresan (Villalba, Hurtado, Guarín y Casas, (2013), nos encontramos en un ambiente dinámico, turbulento y amenazante, y por tanto resulta necesario fomentar este tipo de innovaciones en las escuelas, la cual tiene una importante función, que radica en facilitar los procesos de enseñanza y lograr la integración de los procesos pedagógicos con la realidad latente de las intuiciones y de la comunidad en general; aportando a la solución de problemas de rendimiento académico y deserción estudiantil (Salinas, 2004).

Las instituciones educativas deben tener en cuenta, que como cualquier proceso de innovación educativa, en su etapa de implementación se presentaran una serie de cambios a nivel interno en las que intervendrán factores ideológicos, culturales, psicológicos y sociales, que influirán directamente en el acople y aprehensión de esta innovación en el contexto escolar; sin embargo, con es- tas experiencias se podrá avanzar en el conocimiento pedagógico (Maculan, Jiménez \& Castellanos, 2015); pues estos cambios involucran a todos los actores educativos y requiere la participación activa de los mismos para su éxito, de ahí, la importancia de que toda la comunidad educativa puede aportar, trabajar y apropiarse de los cambios propuestos por la institución para lograr una verdadera transformación e innovación educativa (Salinas, 2004).

La incorporación de nuevos materiales, comportamientos, creencias, concepciones y nuevas prácticas de enseñanzas hacen parte de un proceso de innovación que podría mejorar los procesos de enseñanza-aprendizaje. De esta manera se puede decir que un proceso de innovación pedagógica está basado en la creación de las condiciones para desarrollar la capacidad de aprender y adaptarse en entornos educativo Salinas (2004). Al respecto, Vallejo (2011) puntualiza la necesidad de desarrollar procesos de adaptación que integren un componente personal centrado en valores, actitudes con el desarrollo de tareas asociadas a la formación integral del estudiante.

Desde esta perspectiva, la innovación asociada a los procesos de adaptación puede ser fortalecida a través de la integración de un componente tecnológico en los procesos académicos. Es relevante señalar que son diversos los estudios que realizado sobre la implementación de las tecnologías de la información y la comunicación denominadas en adelante TIC en el contexto educativo, los cuales destacan la importancia de contar con condiciones de infraestructura, disposición de la institución y del cuerpo docente, así como una buena formación e interés de estos para lograr una adecuada y exitosa articulación de las TIC en el proceso de enseñanza; pues el docente 
debe estar en la capacidad de preparar al estudiante para enfrentarse a las exigencias del mundo actual, renovando y actualizando los contenidos curriculares, las habilidades y las competencias que deben ser desarrolladas, coherentes con los nuevos retos de la sociedad actual; es decir, preparar estudiantes con una habilidades de manejo de información, búsqueda, indagación, resolución de problemas, pensamiento crítico, innovación, y autonomía en su propio proceso de formación (UNESCO, 2013).

Es pertinente señalar, que una de las herramientas tecnológicas que influye directamente en la trasformación e innovación en el proceso de enseñanza y despierta el interés de los jóvenes por aprender, por asistir a la escuela y por ende, mejorar su promedio académico, el hacer uso de herramientas WEB; ya que estas permiten resolver problemas y construir competencias y saberes en cualquier momento y lugar; potencializando las destrezas cognitivas, sociales y actitudinales de los estudiantes; pues se aprende de una manera rápida, dinámica e innovadora. (Karam, Buitrago, Fagua y Romero 2013) (Suárez, 2016).

Precisamente al integrarse las nuevas tecnologías al aula de clase, es una manifestación de la necesidad de innovación en la institución; la integración de las TIC permite entonces un cambio en el rol del docente y del estudiante; en el estudiante una oportunidad de adquirir mayor autonomía y responsabilidad en el proceso de aprendizaje; en el docente porque le permite cambiar su metodología tradicional, salir de su roll clásico, e implementar nuevas formas generadoras de conocimiento (UNESCO, 2013).

Es necesario entonces, lograr que los estudiantes se apropien de las TIC y de su utilidad en el entorno educativo y social, desarrollando nuevas capacidades que le permitirán un mejor desempeño en su etapa académica, que logren desarrollar habilidades de comunicación, un pensamiento crítico y flexible, pues como señala Avendaño y Martínez (2013), se ha demostrado que la implementación de estas como herramientas pedagógica, genera mayores intereses en los estudiantes para realizar cualquier actividad curricular.

Es preciso señalar que la problemática que afecta a la Institución Educativa Distrital (IED) Fundación, en los estudiantes del grado octavos desde aproximadamente hace 4 años, radica en la apatía, desmotivación, bajo rendimiento académico y alto nivel de agresividad entre pares; estos problemas generalmente son el resultado de la carencia de estrategias que impacten y generen cambios en el proceso pedagógico. Por lo cual se hace necesario el diseño e implementación de metodologías orientadas desde el uso de elementos tecnológicos que aporten innovación, dinamismo y transformación; herramientas de fácil acceso y en las que se desenvuelven cotidianamente los estudiantes de la institución, como ( Tablet, computadoras y teléfonos celulares); es necesario resaltar que estos episodios o problemas de conducta y desinterés de los estudiantes, también están ligados el entorno familiar; caracterizados por familias disfuncionales, carentes de normas, autoridad y de acompañamiento en las actividades académicas.

Por lo tanto se hace necesario la implementación de las TIC en la institución educativa, ya que proporcionan la creación de ambientes propicios para el aprendizaje, la reflexión y participación, donde se muestra al estudiante que no solo son una herramienta para el ocio y el esparcimiento, sino un motor de indagación, construcción y búsqueda de la información, que les permitirá no solo desarrollar habilidades tecnológicas si 
no también habilidades investigativas desde la implementación de la Investigación como Estrategia Pedagógica (IEP) que se implementara con el proyecto que están desarrollando por parte de los docentes de la Institución Educativa Departamental Fundación titulado diseño de estrategias pedagógicas para incrementar el interés académico en alumnos de octavo grado IED fundación apoyada en las TIC a través de la IEP.

\section{Investigación como estrategia pedagógica}

Actualmente existen diversas propuestas educativas basadas en la investigación, las cuales tienen como objetivo materializar las acciones que se están empleando en diversas instituciones educativas. Con la implementación de estas, surgen un sin números de propuestas metodológicas que buscan apoyarse en anteriores proyectos para lograr un éxito en la ejecución de sus actuales propuestas. Este es el caso de las pedagogías fundadas en la investigación, a las cuales también se les conoce como teorías basadas en la investigación; donde estas asumen caminos variados con respecto a los paradigmas y corrientes, pues ambas se desarrollan en contextos diferentes. Dentro de estas teorías basadas en la investigación, se encuentran dos tipos de propuestas, la enseñanza por descubrimiento y el programa onda con su propuesta de investigación. Esta última retoma para su desarrollo las perspectivas de la pedagogía critico-liberadora y del enfoque socio-cultural (Mejía y Manjarrés, 2010), y es la que se implementa actualmente en las instituciones del Departamento del Magdalena.

Esta propuesta metodológica, de la investigación como estrategia pedagógica fue desarrollada en Inglaterra y
EEUU en los años ochenta por su precursor Jerome Bruner, luego esta se extendió a España donde fue adoptada por el profesorado de la universidad de Valencia para su aplicación, su punto de partida es lograr que los niños logren obtener la capacidad de reconocer un problema como fuente de información, que logren por sus propios medios y mediante la reflexión de la práctica investigativa construir su propio conocimiento, descubrir la estructura de la realidad, así como teorías, procesos, modelos y producto de la ciencia (Mejía y Manjarrés, 2011).

En la implementación de la investigación como estrategia pedagógica los contenidos educativos se convierten en problemáticas con posible solución, donde el currículo pasa a estructurarse a partir del surgimiento de una serie de preguntas educativas expuestas por los estudiantes, los cuales a partir de su descubrimiento plantean la realidad de la institución, lo tornan flexible y se proyecta a fomentar capacidades de análisis y raciocinios en los estudiantes; ese descubrimiento generalmente no se hace de manera autónoma este es guiado por el acompañamiento del docente que es el que se encarga de planificar las estrategias, los ejercicios y los procedimientos para tal fin (Mejía y Manjarrés, 2010).

En otro orden de ideas se puede decir, que la investigación como estrategia pedagógica se da una estrecha relación entre la práctica pedagógica y la investigación; pues buscan que los niños y niñas logren apropiarse de la lógica del conocimiento, y de herramientas propias de la ciencia, apuntando a brindar soluciones sus necesidades y a la comunidad en general, generando procesos o aprendizajes significativo.

En palabras de Mejía y Manjarrés (2010), la propuesta de la IEP como tal, 
no es formar científicos, si no construir una cultura ciudadana y democrática en ciencia, tecnología e innovación, que se ajusta a los cambios frecuentes en la educación actual, donde se establezcan nuevas formas de adquisición de conocimiento articulando los contenidos curriculares con el uso de nuevas tecnologías, a través de la justicia educativa y la justicia curricular trabajando fuertemente por construir sociedades más justas y menos desiguales.

\section{Metodología}

La investigación se llevó a cabo desde una mirada Cualitativa, bajo el paradigma sociocritico, el cual busca dar una interpretación y comprensión de la realidad de los fenómenos o sucesos que se estudian en su contexto natural y real. El modelo investigativo se orienta desde la investigación acción, la cual se orienta a la práctica educativa y cuyos objetivos principales son la transformación y mejora de una realidad educativa (Cerda, 1997).

\section{Participantes}

La unidad de análisis estuvo constituida por cuarenta (40) estudiantes del grado octavo de bachillerato entre las edades de catorce (14) quince (15) y dieciséis (16). El estudio cumplió con los estándares éticos de solicitud del consentimiento institucional firmado por los padres de los respectivos estudiantes.

Técnicas e instrumentos de recolección y organización de información

Teniendo en cuenta el tipo de investigación se utilizó técnica de recolección de información la observación participante, en la cual, el investigador observa, escucha y participa del fenómeno estudiado en su contexto real (Hernández,
Fernández y Baptista 2012); así mismo se utilizó como instrumento el diario de campo en el cual el investigador anota los acontecimientos observados, reflexiones, puntos de vista, conclusiones, dudas e inquietudes. (Hernández, Fernández y Baptista 2014).

\section{Procedimiento}

Dado el tipo de investigación, las actividades fueron diseñadas de acuerdo a la problemática presente en la institución, donde además de observar, el maestro participó en cada una de las actividades ejecutadas en el aula de clase, en cada una de las asignaturas a cargo de los miembros del grupo de investigación. Estas actividades fueron diseñadas a partir de los intereses de los estudiantes y de acuerdo a las necesidades pedagógicas que estos presentan. El proceso de observación se realizó durante dos semanas, implementando dos actividades por cada una; al final del proceso se logró una interacción positiva entre maestro y estudiantes lo que logró un resultado efectivo en el desarrollo de las actividades. Al final de cada observación de cada una de las actividades, se registraron de todas las situaciones que se presenten durante el desarrollo de la ejecución de las actividades.

\section{Resultados}

A continuación, se presentan los resultados derivados de las actividades ejecutadas en el aula de clase, con la implementación de las TIC como herramientas dinamizadoras para fortalecer el interés académico de los estudiantes. En la realización de estas actividades se observó un cambio significativo en la disposición del estudiante frente a los diferentes temas tratados, tornándose proactivos, interesados y dispuestos a aprender (tabla1). 
TABLA1

Incorporación de las TIC en el aula de clases

\begin{tabular}{ll}
\hline \multicolumn{1}{c}{ Actividad } & \multicolumn{1}{c}{ Descripción } \\
\hline & \\
& \\
& El docente presenta \\
su clase a través del & computador, donde al \\
La computadora & tiempo esta presentación \\
pedagógica. & se proyecta en cada uno de \\
& los computadores.
\end{tabular}

La actividad logró generar un cambio en la metodología tradicional de enseñanza, donde se presentan las actividades académicas a través de herramientas tecnológicas, mostrándole al estudiante las virtudes y ventajas del buen uso de estas en el contexto escolar. Es importante reconocer el papel que desempeñan las TIC en el contexto educativos, en especial el uso del computador dentro del aula de clase, pues este permite articular el currículo, el diseño metodológico, y la teoría en una sola dimensión, que dinamiza y genera mayor impacto en la práctica pedagógica.

La actividad realizada en el aula de clase permitió una movilización activa en la participación de los estudiantes, logró generar un espacio para el

Los estudiantes a través de las tablets buscan noticias o casos del uso

El internet como fuente de información

Trabajo de la mano con la investigación y el internet. debate, la crítica, facilitando el proceso de enseñanza aprendizaje; así mismo, la actividad fomento la investigación, pues se partía de la indagación de un tema específico para luego a través de la práctica lograr una apropiación de los saberes; logrando establecer que el uso del internet no se limita al ocio y la recreación. En este sentido Aguaded y Cabero (2002), sostiene que el internet es una fuente de información inagotable, que facilita y proporciona a gran cantidad de instrumentos y técnicas para el desarrollo de las clases; favoreciendo el trabajo cooperativo, autonomía, responsabilidad y motivación por el aprendizaje.

Los resultados de la actividad, permitió establecer grupos de investigación en el aula de clase, se fomentó el buen uso de las herramientas tecnológicas, se resolvieron preguntas de la cotidianidad del contexto escolar, articulando las investigación con todas las áreas curriculares; los estudiantes comprendieron la importancia de la infinidad de información que posee el internet y su aplicabilidad en la investigación. Se destaca entonces la importancia de las TIC en la investigación como plantean Corredor \& Sandino, (2009), quienes sostiene que el uso de las TIC en la investigación científica se evidencia en las múltiples indagaciones que el investigador realiza en los diversos buscadores de internet, así como también en la participación e interacción con diferentes comunidades de aprendizaje y foros virtuales, conformando lo que se denomina una comunidad científica, facilitándose el intercambio de información para la construcción social de conocimientos. Autores como Gutiérrez, Carrera, Marín, Narváez y Pérez (2006), asocian las comunidades de aprendizaje con la conformación de redes académicas como base para la integración de comunidades científicas que contribuyan al desarrollo. 


\begin{tabular}{|c|c|c|}
\hline $\begin{array}{l}\text { Las redes } \\
\text { sociales como } \\
\text { herramientas } \\
\text { para fomentar el } \\
\text { interés académico } \\
\text { de los estudiantes }\end{array}$ & $\begin{array}{l}\text { Se utilizaron las redes } \\
\text { sociales en el aula de clase } \\
\text { y fuera de ella, para enviar } \\
\text { y compartir información } \\
\text { relevante del contexto } \\
\text { escolar; los estudiantes } \\
\text { crearon grupos de difusión } \\
\text { por WhatsApp, correo } \\
\text { electrónico escolar y } \\
\text { páginas en facebook, } \\
\text { donde debían compartían } \\
\text { la información enviada } \\
\text { por el docente, ampliando } \\
\text { el contenido de la misma } \\
\text { a través de la búsqueda } \\
\text { por internet, finalmente } \\
\text { compartían dicha } \\
\text { información a través de } \\
\text { las mismas con toda la } \\
\text { comunidad educativa }\end{array}$ & $\begin{array}{l}\text { Se implementaron las redes sociales (correo } \\
\text { electrónico, facebook y whatsApp), como herramientas } \\
\text { de intercambio de información, búsqueda y } \\
\text { socialización de resultados; se evidencio el interés de } \\
\text { los estudiantes por participar, compartir información } \\
\text { y asistir a las clases, mejorando significativamente las } \\
\text { notas provenientes de estas actividades. El uso de las } \\
\text { redes sociales en el aula de clase y fuera de ella como } \\
\text { herramienta educativa, logra generar espacios para la } \\
\text { interacción participación y descubrimiento, donde se } \\
\text { materializan las relaciones amenas entre estudiantes } \\
\text { y profesores, se dinamiza la actividad curricular y } \\
\text { se generan espacios de intercambio de información, } \\
\text { desarrollando así, la producción y socialización del } \\
\text { conocimiento. }\end{array}$ \\
\hline
\end{tabular}

Fuente: elaboración propia.

Los resultados arrojados en cada una de las actividades dejan en evidencia la importancia de articular los contenidos curriculares y la praxis del docente, con herramientas tecnológicas que innoven y dinamicen el proceso de enseñanza- aprendizaje, donde se constituyan comunidades de saber y conocimiento en torno a las necesidad de las institución, orientadas a la trasformación pedagógica, a fortalecer las competencias científicas de los estudiantes, sus interés y a prevenir situaciones problema en torno a la desmotivación y ausentismo escolar por la falta de estrategias al interior del aula de clases. Es entonces relevante, que el docente adquiera las competencias necesarias para enfrentarse a los nuevos retos de la educación actual, sumergida en cambios que apuntan a la consolidación de una educación integral, que avanza y genera facilidad en la adquisición de los saberes (Hernández, 2017).

En torno a que la actividad pedagógica generalmente se presenta de manera presencial, es necesario acompañar dicho proceso con el uso de la tecnología; dando un giro trascendental a la pedagogía tradicional, para trabajar con elementos innovadores, que impulsen al estudiante a enfrentarse a los retos de la sociedad actual, que se aleja de un aprendizaje pasivo de memorización; enmarcándolo en una era de un aprendizaje reflexivo, que cuestiona, critica y propone desde su propia experiencia (Cortes et al., 2015)

Es necesario destacar que al hablar de la incorporación de las TIC en la educación, no solo se enfatiza en el uso de computadores y herramientas digitales; se habla de crear ambientes educativos propicios para la construcción de conocimiento, de consolidar un aprendizaje significativo, que propicia la consolidación de comunidades de aprendizaje, donde se interactúa con estas, como herramientas de carácter pedagógico (Díaz y Barriga, 2013). Así mismo, tal como señala Avendaño y Martínez (2013), el uso frecuente de estas herramientas pedagógicas en el contexto escolar genera cambios en la percepción del estudiante, incrementando sus niveles de motivación y participación en la realización de s actividades académicas de cualquier tipo de contenido. 


\section{Discusión}

Conforme a los resultados obtenidos en la investigación dando respuesta al objetivo planteado se concluye que es pertinente emplear diversas estrategias pedagógicas mediante el uso de las TIC, que potencialicen el incremento del interés académico de los estudiantes de octavo grado de la IED Fundación, aportando de esa manera a dinamizar y diversificar los estilos de enseñanzaaprendizaje.

Se requiere por medio del uso de las TIC como estrategia pedagógica empoderar a maestros y estudiantes para que asuman un rol activo en el proceso de enseñanza aprendizaje, que no se limiten a la información que les ofrece el entorno escolar y accedan a la multiplicidad de información que se aloja en la internet, y que a través de los proceso de búsqueda e indagación se potencialicen competencias investigativas y científicas.

No obstante para que pueda haber un verdadero impacto de las TIC en las metodologías y procesos de enseñanza aprendizaje, se requiere un trabajo cooperativo entre de las políticas institucionales, la administración escolar, docentes y actores involucrados, para que se fomente la construcción de un proyecto educativo claramente diseñado, flexible, y compartido como señala Castro, Guzmán, Casado (2007). Teniendo en cuenta lo anterior dicho, es necesario que todos los actores que involucran el contexto escolar, comprendan y asuman su papel en la implementación de las herramientas tecnológicas en el ámbito escolar, pues si no trabajan articuladamente no se evidenciara su impacto en los niveles de educación. Herrera (2016).
Cabe señalar que en la ejecución de las actividades diseñadas y ejecutas en la presente investigación, se logró evidenciar el empoderamiento de los docentes en el proceso de reconocimiento y apropiación de nuevas metodologías a emplear en el aula de clases; abriéndose a generar un cambio en la metodología tradicional que los ha acompañado desde hace muchos años, para entrar en una era mediada por las TIC, que impacte y logre cambios significativos en la educación de los estudiantes.

Finalmente se logró constatar altos niveles de motivación e interés de los estudiantes con la implementación de las actividades, evidenciándose en el desempeño académico y su participación activa durante el desarrollo de las clases; corroborando lo planteado por Ospina (2013), quien sostiene que al trabajar con herramientas tecnológicas el grado de motivación de los estudiantes incrementa, pues las herramientas se tornan novedosas e interesantes, que generan un ambiente de aprendizaje cómodo, colaborativo y propicio para el aprendizaje.

\section{Referencias}

Aguaded, J. y Cabero, J. (2002): Educar en Red. Internet como recurso para la educación. Málaga: Aljibe

Avendaño, I. y Martínez, D. (2013). Competencia lectora y el uso de las nuevas tecnologías de la información y comunicación. Revista Escenarios, 11(1), 7-22.

Castro, S., Guzmán, B. y Casado, D. (2007). Las TIC en los procesos de enseñanza y aprendizaje. Laurus, 13(23). 213-234.

Cerda, H. (1997). La investigación total. Bogotá, D.C.: Cooperativa Editorial Magisterio. 
Corredor, A. y Sandino, M. (2009). Las TIC como herramienta de investigación científica. Góndola, enseñanza y aprendizaje de las ciencias, 4(1), 2529.

Martínez, J., López, G., Rodríguez, V. y Martínez, A. (2015). El Aprendizaje Distribuido, primer pasó a la virtualidad en la Enseñanza en el Nivel Universitario. Revista Iberoamericana de Producción Académica y Gestión Educativa, 2(3). 1-14. Recuperado de https://www.pag.org.mx/index. php/PAG/article/viewFile/263/309

Díaz, F. (2013). TIC en el trabajo del aula. Impacto en la planeación didáctica. Revista Iberoamericana de Educación Superior, 4(10), 3-21.

Gutiérrez, C., Carrera, M., Marín, F., Narváez, M. y Pérez, C. (2006). Integración de Redes Académicas para la Gestión del Desarrollo Endógeno Regional. Multiciencias 6(3), 257-263.

Hernández, R. (2017). Impacto de las TIC en la educación: Retos y Perspectivas. Propósitos y Representaciones, 5(1), 325-347. Extraído de http:// dx.doi.org/10.20511/pyr 2017 . v5n1.149

Hernández, R., Fernández, C. y Baptista, P. (2014). Metodología de la investigación. México, D.F.: McGraw-Hill Education.

Karam, J., Buitrago, A., Fagua, A. y Romero, Y. (2013). Estrategias de gamificación aplicadas al diagnóstico de la incorporación pedagógica de las TIC en una comunidad académica. Cultura, Educación y Sociedad 4(1), 55-74.

Maculan, A., Jiménez, C. y Castellanos, O. (2015). Aprendizaje en el proceso de incubación de empresas de base tecnológica. Económicas CUC, 36(1), $15-48$.
Marín, F., Inciarte, A., Hernández, H. y Pitre, R. (2017). Estrategias de las Instituciones de Educación Superior para la Integración de las Tecnología de la Información y la Comunicación y de la Innovación en los Procesos de Enseñanza. Un Estudio en el Distrito de Barranquilla, Colombia. Formación universitaria, 10(6), 29-38.

Mejía, M. y Manjarrés, M. (2011). La investigación como estrategia pedagógica una apuesta por construir pedagogías críticas en el siglo XXI. Praxis \& Saber, 2(4), 127. http:// dx.doi.org/10.19053/22160159.1127

Mejía, M. y Manjarrés, M. (2010). Las pedagogías fundadas en la investigación. Búsquedas en la reconfiguración de la educación. Revista Internacional Magisterio, 42(1). 16 26.

Ospina, C. (2013). Las TICS como herramienta de motivación en el aula: Una investigación sobre la integración de las TIC en educación. [Tesis de maestría]. Universidad de La Sabana, Bogotá, D.C., Colombia.

Salinas, J. (2004). Innovación docente y uso de las TIC en la enseñanza universitaria. Revista Universidad y Sociedad del Conocimiento. RUSC, 1(1), 1-16.

Suárez, O. (2016). Aproximación al origen de la noción de objeto de aprendizaje: revisión histórico-bibliográfica. INGE CUC, 12(2), 26-40. https://doi. org/10.17981/ingecuc.12.2.2016.03

UNESCO. (2013). Enfoques estratégicos sobre las Tics en educación en América latina y el caribe. Recuperado de http://www.unesco.org/new/ fileadmin/MULTIMEDIA/FIELD/ Santiago/images/ticsesp.pdf 
Vallejo, O. (2011). Adaptación laboral: Factor clave para el rendimiento y la satisfacción en el trabajo. Cultura Educación y Sociedad 2(1), 171 - 176. Recuperado de https://revistascientificas. cuc.edu.co/culturaeducacionysociedad/ article/view/946
Villalba, M., Hurtado, H., Guarín, H. y Casas, J. (2013). Innovación en pymes artesanales de Morroa, Sucre, 2012. Económicas CUC, 34(1), 15-28. 